\title{
An Energy-Scalable In-Band Full Duplex Architecture
}

\author{
Tom Vermeulen*, Fernando Rosas*, Barend van Liempd ${ }^{\dagger \ddagger}$, Marian Verhelst* and Sofie Pollin* \\ ${ }^{*}$ Department of Electrical Engineering, KU Leuven, Heverlee B-3001 Belgium \\ $\dagger_{\text {imec, }}$ Belgium

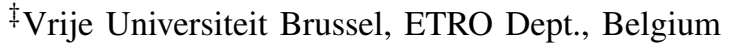

\begin{abstract}
In-band full duplex (IBFD) is a promising technique that allows to potentially double the achievable bi-directional throughput over a given bandwidth. Moreover, it has been estimated that IBFD-equipped wireless networks can be more energy-efficient than half duplex ones due to the reduced energy cost of packet collisions. However, one key challenge for implementing an energy-efficient IBFD system is the cancellation of the interference produced by the transmitted signal. In fact, existing self-interference cancellation (SIC) techniques are not always energy-efficient, as the effect of the interference reduction might not be able to compensate the additional electronic power consumption introduced by the SIC module.

Following this rationale, in this paper we propose and energyefficient IBFD architecture that adapts the SIC module to the link conditions. By studying a symmetric bi-directional full duplex link, we show that the proposed architecture obtains significant energy savings by using a simple SIC scheme for short-range transmissions. More powerful SIC techniques are shown to be an energy-optimal choice only when transmitting over long link distances.
\end{abstract}

\section{INTRODUCTION}

One of the main challenges for next-generation wireless systems will be to provide high data rates at a low energy cost [1]. Achieving this higher throughput by increasing the bandwidth would not be a realistic option, as spectrum is already crowded and the number of wireless devices is rapidly increasing [2]. One solution for achieving the much desired additional bandwidth is to look for additional spectrum in higher frequencies [3]. Another solution, complementary with the previous one, is to look for additional degrees of freedom to increase the spectral efficiency, allowing to achieve a higher throughput without increasing the bandwidth requirements.

An interesting technique that falls into the second category is in-band full duplex (IBFD), which allows wireless nodes to simultaneously transmit and receive data on the same frequency. IBFD enables to potentially double the bi-directional throughput without increasing the spectrum usage [4]. Interestingly, it has recently been shown that in-band full duplex technologies can also increase the energy efficiency of nodes in congested wireless networks [5]. In particular, [5] shows that IBFD-equipped IEEE 802.15.4 nodes are more energyefficient than regular half duplex nodes, as a full duplex MAC scheme is able to mitigate the energy cost of collisions.

One of the key challenges to implement IBFD, however, is the cancellation of the self-interference (SI) produced by the transmitted signal, which can be various orders of magnitude larger than the received signal. Several self-interference cancellation (SIC) schemes have been proposed in the literature, which can be combined in order to achieve an improved cancellation of the SI signal. However, each of these SIC schemes consume additional power for tuning and activating dedicated electrical components. Therefore, from an energy efficiency point of view, it is not clear if the gains that the SIC schemes provide on the signal-to-self-interference-plusnoise ratio (SSINR) are worth the increased electronic power consumption of the transceiver.

For addressing this issue, we propose a scalable IBFD architecture that explores the trade-off between SIC and electronic power consumption for achieving a higher energy efficiency. The proposed architecture contains three SIC schemes, which can be selectively activated in function of the link conditions. The key intuition behind our proposal is the fact that it is not always beneficial in terms of energy efficiency to use the most expensive SIC scheme available. In fact, such SIC scheme could consume a lot of power to cancel interference even when it is already well below the noise floor.

To analyze the proposed IBFD architecture, we study its performance on a symmetric bi-directional IBFD link using a detailed energy consumption model. In this way, we explore the trade-off that exists between the reduction of SI and the increased power consumption, finding its optimal balance in terms of the overall energy budget. Our results show that our architecture can obtain significant energy savings by using a simple SIC scheme for short link distances, as the reduced path loss enables to achieve a high SSINR even with low SIC levels. On the other hand, complex SIC schemes are better suited for long range transmissions, as a strong SIC allows to reduce the number of transmission trials required to obtain a correctly decoded frame.

The rest of this paper is organized as follows. First, Section II discusses the proposed SIC architecture and explains the link budget trade-offs. Then, Section III presents the performance model, which is later evaluated in Section IV. Finally, Section V contains our main conclusions.

\section{SCAlable FUll DUPleX ARCHITECTURE}

A scalable IBFD architecture should be able combine and selectively (de)activate distinct SIC components to optimize energy efficiency. Thanks to this scalability, the system is able to find a good balance between SI cancellation and electronic 
power consumption. In the following, Section II-A presents an overview of different SIC solutions. Then, Section II-B describes our proposed scalable IBFD architecture, and Section II-C explains the possible trade-offs in the link budget.

\section{A. Overview of in-band full duplex SIC solutions}

With in-band full duplex transceivers, each SIC module uses the known transmitted signal as a reference to cancel the SI signal from the received signal. Although the transmitted signal is known, non-idealities of the circuits make this cancellation far from trivial, specially due to the fact that the transmitted signal is various orders of magnitudes above the received signal. In fact, usually a number of cancellation stages are combined sequentially in order to achieve a good cancellation performance.

In the literature, researchers have proposed several SIC schemes. In [6] an electrical balance duplexer (EBD) is presented, which balances the impedance from the antenna in order to create an inverse copy of the SI signal. This inverse copy destructively interferes with the SI signal, achieving a cancellation of at least $50 \mathrm{~dB}$ at RF. Another SIC technique is the vector modulator $(\mathrm{VM})$ scheme, which consist of a second downconverter that creates a delayed and scaled version of the SI signal which is later subtracted from the main signal [7]. It has been shown that this scheme is able to cancel the SI signal for at least $20 \mathrm{~dB}$ at RF. Other RF solutions use a noise canceler chip [8] or an additional transmitter to create a canceling signal [9].

Besides in RF, cancelation can also be performed in the digital domain. In this case, the remaining self-interference is canceled by estimating the SI channel, recreating the SI signal and subtracting it [8]. This requires enough dynamic range from the analog-to-digital converter, meaning that it can only be done when sufficient RF cancellation is provided. This type of cancellation is able to provide at least $30 \mathrm{~dB}$ of SIC.

\section{B. Proposed architecture}

A useful indicator of the quality of the signal after the SIC module is the mean signal-to-self-interference-plus-noise ratio (SSINR), $\bar{\gamma}$, which is defined as

$$
\bar{\gamma}=\frac{P_{\mathrm{rx}}}{g_{\mathrm{SIC}} P_{\mathrm{tx}}+P_{\mathrm{n}}} .
$$

Above, $P_{\mathrm{rx}}$ and $P_{\mathrm{tx}}$ are the average received and transmitted signal power, respectively, $P_{\mathrm{n}}$ is the average thermal noise power and $1 \geq g_{\mathrm{SIC}} \geq 0$ is the cancellation performance of the SIC schemes. As a first approach, we assume that the remaining self-interference can be modeled as additive white Gaussian noise. If the cancellation performance is such that $g_{\mathrm{SIC}} \gg P_{\mathrm{n}} / P_{\mathrm{tx}}$, then (1) to

$$
\bar{\gamma} \approx \frac{P_{\mathrm{rx}}}{g_{\mathrm{SIC}} P_{\mathrm{tx}}}=\frac{\Lambda}{g_{\mathrm{SIC}}},
$$

where $\Lambda=P_{\mathrm{rx}} / P_{\mathrm{tx}}$ is the link path loss. Note that, under this condition, variations in the radiated power do not impact the SSINR as the increase in received signal power is nullified by a corresponding increase in the SI.

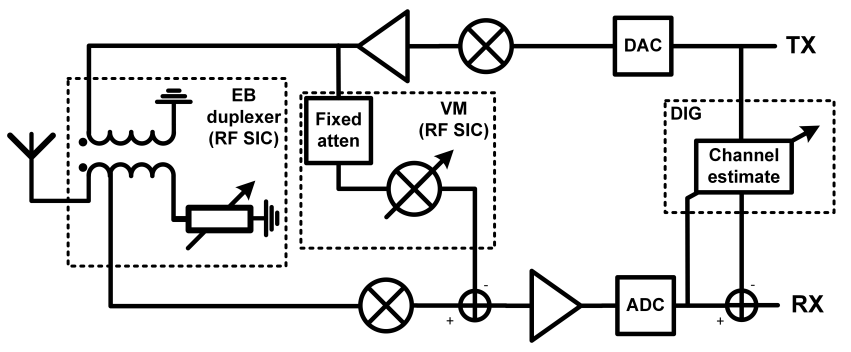

Fig. 1. Proposed scalable full duplex architecture

To achieve enough $g_{\text {SIC }}$, we propose a scalable IBFD architecture that uses three successive SIC stages. The architecture has been designed in a way that each module can be independently (de)activated (see Figure 1). First, in the RF domain, our system uses an EBD to first cancel the SI signal. Next, still in the RF domain, a VM is used to cancel the signal even further. After this, the signal is converted to the digital domain, where a digital FIR-based SIC cancels the remaining self-interference. In our design, it is possible to turn off the vector modulator as well as the digital cancellation. This gives four different combinations which we investigate: (1) EBD + VM + DIG, (2) EBD + VM, (3) EBD + DIG and (4) EBD only. Table I gives an overview of the cancellation performance of these SIC schemes. Because of real-world experience with the devices, we have found that these numbers are additive, meaning that combined they can achieve a $-100 \mathrm{~dB}$ cancellation [10].

TABLE I

OVERVIEW OF SIC COMBINATIONS

\begin{tabular}{|c|c|}
\hline \hline Combination & Cancellation performance $\left(g_{\text {SIC }}\right)$ \\
\hline \hline EBD + VM + DIG & $-100 \mathrm{~dB}$ \\
EBD + VM & $-70 \mathrm{~dB}$ \\
EBD + DIG & $-80 \mathrm{~dB}$ \\
EBD & $-50 \mathrm{~dB}$ \\
\hline
\end{tabular}

\section{Full duplex link budget}

When designing an in-band full duplex system, most designers target to cancel the self-interference until its power is below the noise floor in order to keep the same SNR as a half duplex link [4]. Nevertheless, it is interesting to explore if less SIC could be optimal from an energy efficiency point of view. For example, consider a received power of $-70 \mathrm{dBm}$ and a transmit power of $0 \mathrm{dBm}$. If the system has a noise power of $-90 \mathrm{dBm}$ as presented in Figure 2, then two out of three of the cancellation schemes could be enough to cancel the SI signal sufficiently. The combination of an EBD and digital cancellation give a SSINR of $10 \mathrm{~dB}$ compared to a SSINR of $20 \mathrm{~dB}$ if one were to cancel it below the noise floor. Using only two cancellation schemes saves energy in cancellation but could potentially increase the number of retransmissions due to decoding errors. Furthermore, for a transmit power of $-20 \mathrm{dBm}$, the remaining SI power is at $-120 \mathrm{dBm}$, far below 


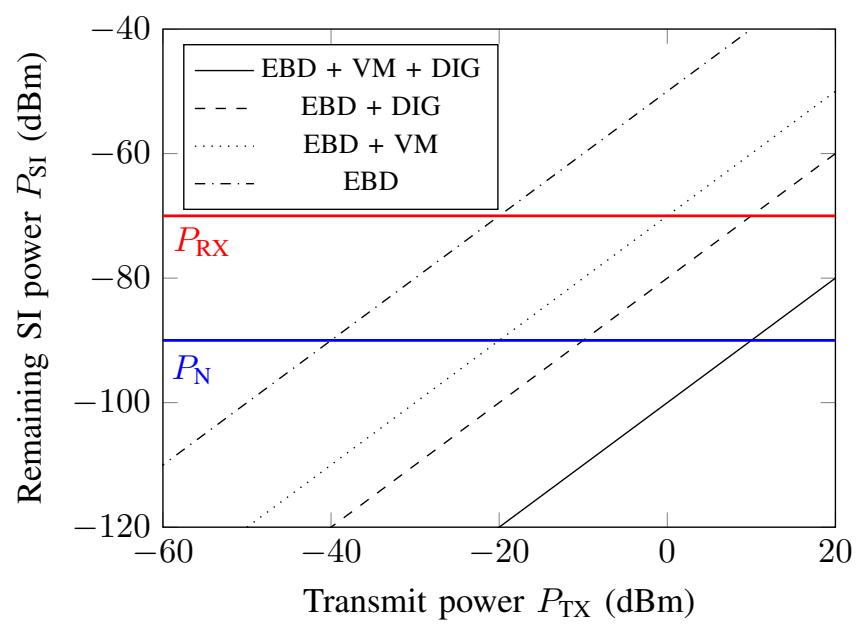

Fig. 2. The remaining SI power after cancellation as function of the transmission power, assuming a noise floor of $-90 \mathrm{dBm}$ and received power of $-70 \mathrm{dBm}$

the noise power. Again two cancellation schemes should be enough, as self-interference cancellation far below the noise floor is not necessary. These trade-offs are investigated in the remaining of this paper.

\section{PERFORMANCE MODEL}

In this section we determine the total amount of energy that is required to successfully transmit one bit of data using the proposed IBFD architecture. First, Section III-A discusses the energy consumption of the SIC modules, and then Section III-B presents our full duplex energy consumption model.

\section{A. Energy consumption SIC}

The energy consumption of the EBD per data bit per transmission trial is given by

$$
\mathcal{E}_{\mathrm{EBD}}=\frac{P_{\mu \mathrm{C}} T_{\mathrm{EBD}}}{r L_{\mathrm{p}}}
$$

where $P_{\mu \mathrm{C}}$ is the power consumed by the microprocessor and $T_{\mathrm{EBD}}$ is the time it takes the algorithm to find an optimal working point for the EBD. Note that the EBD is a passive component, and hence power is only consumed for tuning the balance network. No additional power is required during the frame transmission. Let us define $r=k / n$ as the code rate, where $n$ is the number of bits per codeword and $n-k$ is the number of added redundancy bits. Therefore, there are $r L_{\mathrm{P}}$ data bits per frame, with $L_{\mathrm{P}}$ the payload size in bits.

By denoting the time to tune the $\mathrm{VM}$ as $T_{\mathrm{VM}}$, the energy consumption per data bit for the VM can be modeled as,

$$
\mathcal{E}_{\mathrm{VM}}=\frac{P_{\mu \mathrm{C}} T_{\mathrm{VM}}}{r L_{\mathrm{p}}}+P_{\mathrm{VM}} T_{\mathrm{b}} .
$$

This device is also passive, but requires $P_{\mathrm{VM}}$ watts of power to drive the LO buffers for the mixer during the cancellation of the whole frame transmission. The amount of time per bit that the mixer needs to be active is denoted by $T_{\mathrm{b}}$, which corresponds to the air time per payload bit (c.f. (8)).
Finally, the energy consumption per data bit for the digital cancellation is given by

$$
\mathcal{E}_{\mathrm{DIG}}=P_{\mathrm{FIR}}\left(\frac{T_{\mathrm{FIR}}}{r L_{\mathrm{p}}}+T_{\mathrm{b}}\right)
$$

where $P_{\text {FIR }}$ is the power consumption of the FIR filter and $T_{\text {FIR }}$ is the time it takes to estimate the channel. As with the VM, digital cancellation consumes power both during the control loop and the cancellation that takes place during the frame transmission, causing the added term $\left(T_{\mathrm{b}}\right)$.

The energy per bit for the full SIC scheme of our scalable architecture is given by

$$
\mathcal{E}_{\mathrm{SIC}}=\chi_{1} \mathcal{E}_{\mathrm{EBD}}+\chi_{2} \mathcal{E}_{\mathrm{VM}}+\chi_{3} \mathcal{E}_{\mathrm{DIG}}
$$

where $\chi_{1}, \chi_{2}$ and $\chi_{3}$ are indicator variables which are equal to 1 if the corresponding module is active and 0 if it's not. Based on our experience with the devices, we assume these SIC components need to be retuned every packet, as impedance variations of the antenna change the optimal working point of the components.

\section{B. Full duplex energy consumption}

We focus on point-to-point transmissions between two low power devices equipped with the proposed IBFD architecture. We consider packet-switched transmissions, where it is assumed that all frames are always detected. Under these assumptions, the total energy required by a node for sending one bit of data successfully can be expressed as

$$
\overline{\mathcal{E}}_{\mathrm{b}}^{\mathrm{FD}}=\left[\left(P_{\mathrm{el}, \mathrm{tx}}+P_{\mathrm{PA}}+\alpha P_{\mathrm{el}, \mathrm{rx}}\right) T_{\mathrm{b}}+\mathcal{E}_{\mathrm{SIC}}\right] \bar{\tau} .
$$

Above, $P_{\mathrm{PA}}$ is the power consumed by the power amplifier (PA) and $P_{\mathrm{el}, \mathrm{tx}}\left(P_{\mathrm{el}, \mathrm{rx}}\right)$ is the total power consumed by the remaining baseband and radio-frequency electronic components required for transmitting (receiving) a frame. The parameter $0<\alpha<1$ accounts for electronic components that are shared between transmitter and receiver. Finally, $\bar{\tau}$ is the average number of transmission trials required until a frame is decoded without errors in the receiver, which depends on PHY parameters such as the channel statistics, SNR and modulation, and on link layer parameters such as code rate and frame size.

To find an explicit expression for $T_{\mathrm{b}}$, we consider a physicallayer frame that carries $L_{\mathrm{H}}$ bits of header and a payload composed by $r L_{\mathrm{P}}$ bits of data and $(1-r) L_{\mathrm{P}}$ additional bits for coding. The total duration of a frame is shared by $T_{\mathrm{P}}$ seconds for transmitting the payload, $T_{\mathrm{H}}$ seconds for transmitting the header and $T_{\mathrm{O}}$ seconds for the transmission of overhead signals for acquisition and tracking (channel estimation, synchronization, etc.). The air time per data bit in a frame is $T_{\mathrm{b}}=\frac{T_{\mathrm{P}}+T_{\mathrm{H}}+T_{\mathrm{O}}}{r L_{\mathrm{P}}}$. Let us define $R_{\mathrm{s}}$ as the physical layer symbol rate and $b=\log _{2} M$ as the number of bits per symbol. By noting that $L_{\mathrm{P}} / T_{\mathrm{P}}=b R_{\mathrm{s}}$, one can express $T_{\mathrm{b}}$ as

$$
T_{\mathrm{b}}=\frac{1}{r R_{\mathrm{s}}}\left(\frac{1}{b}+\frac{L_{\mathrm{H}}}{b L_{\mathrm{P}}}+\frac{L_{\mathrm{O}}}{b L_{\mathrm{P}}}\right),
$$

where $L_{\mathrm{O}}=T_{\mathrm{O}} / b R_{\mathrm{s}}$ is the total overhead measured in bits. This overhead is dependent on the chosen SIC schemes as 
all require some extra symbols to find their optimal working point. This means that the transmitter and receiver need to be active during tuning, which is incorporated in $T_{\mathrm{b}}$. The EBD, VM and DIG add 4,2 and 4 bytes in overhead respectively.

The number of transmission trials until a frame is decoded without error, $\tau$, is a random variable whose statistics have been studied in [11]. Under the assumption that the transmitter is equipped with a deep interleaver, it can be shown that [11, eq. (9)]

$$
\bar{\tau}=\frac{1}{1-\mathbb{E}\left\{P_{\mathrm{f}}\right\}},
$$

where $\mathbb{E}\left\{P_{\mathrm{f}}\right\}$ is the mean frame error rate. Consistent with the IEEE 802.15.4 standard, the rest of this article focuses on the case of uncoded communications - although an extension of our results for the case of coded transmissions could be developed using the results presented in [12]. Then $\mathbb{E}\left\{P_{\mathrm{f}}\right\}$ can be expressed in terms of the bit error rate of the $M$-ary modulation $P_{\mathrm{b}}(\gamma)$ and the binary modulation symbol error rate $P_{\text {bin }}(\gamma)$ as

$$
\bar{P}_{\mathrm{f}}(\bar{\gamma})=1-\left[1-\bar{P}_{\mathrm{bin}}(\bar{\gamma})\right]^{L_{\mathrm{H}}}\left[1-\bar{P}_{\mathrm{b}}(\bar{\gamma})\right]^{L_{\mathrm{P}}},
$$

where $\bar{P}_{\text {bin }}(\bar{\gamma})=\mathbb{E}\left\{P_{\text {bin }}(\gamma)\right\}$ and $\bar{P}_{\mathrm{b}}(\bar{\gamma})=\mathbb{E}\left\{P_{\mathrm{b}}(\gamma)\right\}$ with the expected value calculated over a given channel fading distribution. Above, $\bar{\gamma}$ corresponds to the mean SSINR as defined by (1).

\section{RESUlts}

This section presents the results of numerical evaluations of the energy consumption model presented in Section III. For this, we studied (7) for different link distances using parameters of the IEEE 802.15.4 standard (see Table II). Both $T_{\mathrm{EBD}}$ and $T_{\mathrm{VM}}$ were estimated from real-world experience with the corresponding devices, and the FIR power consumption was calculated from [13] using a 4-tap 10 bit filter in $90 \mathrm{~nm}$ technology.

For each transmission distance and each SIC setup, the radiated power has been selected as follows. Following the datasheet of the TI CC2420 transceiver [14], we considered a discrete set of eight radiation power levels ranging from -25 to $0 \mathrm{dBm}^{1}$. The corresponding received power is calculated using a path loss given by the Friis equation:

$$
\Lambda=\frac{P_{\mathrm{rx}}}{P_{\mathrm{tx}}}=\left(\frac{\lambda}{4 \pi D}\right)^{2},
$$

where $D$ denotes the link distance and $\lambda$ the wavelength of the carrier frequency. Then, the mean frame error rate is computed by evaluating (10) using a SSINR as given by , and considering, for the received useful signal, Rician channel fading statistics with 30 times more power in the line-of-sight than in the scattered signal components. Then, the overall energy consumption is computed using (7) and the corresponding $\bar{\tau}$ given by (9). Finally, after exploring the eight radiation power levels, the transmitter chooses the one

\footnotetext{
${ }^{1}$ Note that Table II only shows the power consumption for $0 \mathrm{dBm}$.
}

TABLE II

PARAMETERS USED FOR NUMERICAL EVALUATIONS

\begin{tabular}{|c|c|}
\hline \hline Parameter & IEEE 802.15 .4 \\
\hline Frame Header $-L_{\mathrm{H}}$ & 1 byte \\
Payload length $-L_{\mathrm{P}}$ & 127 bytes \\
Overhead $-L_{\mathrm{O}}$ & $5-11$ bytes \\
\hline Bit per symbol $-b$ & 2 \\
Symbol rate $-R_{\mathrm{S}}$ & $125 \mathrm{kS} / \mathrm{s}$ \\
Wavelength $-\lambda$ & $125 \mathrm{~mm}$ \\
\hline Tx electronic power (0dBm) $-P_{\mathrm{el}, \mathrm{tx}}+P_{\mathrm{PA}}$ & $30.67 \mathrm{~mW}^{*}$ \\
Rx electronic power $-P_{\mathrm{el}, \mathrm{rx}}$ & $35.28 \mathrm{~mW}^{*}$ \\
Full duplex power ratio $-\alpha$ & $0.7449^{\dagger}$ \\
Microprocessor power- $P_{\mu \mathrm{C}}$ & $13.53 \mathrm{~mW}^{\S}$ \\
VM power $-P_{\mathrm{VM}}$ & $7 \mathrm{~mW}^{\ddagger}$ \\
FIR power $-P_{\mathrm{FIR}}$ & $200 \mu \mathrm{W}^{\prime \prime}$ \\
\hline EBD control time $-T_{\mathrm{EBD}}$ & $128 \mu \mathrm{s}$ \\
VM control time $-T_{\mathrm{VM}}$ & $64 \mu \mathrm{s}$ \\
FIR control time $-T_{\mathrm{FIR}}$ & $128 \mu \mathrm{s}$ \\
\hline
\end{tabular}

Source: ${ }^{\dagger}$ [5], ${ }^{\ddagger}[7],{ }^{11}[13],{ }^{*}[14], \S[15]$.

that minimizes (7), which corresponds to the optimal radiation power for that link distance.

Results show that, for short distances, our architecture is able to achieve significant energy savings by turning off the VM and/or the DIG (see Figure 3). The low path loss due to the short range allows to achieve a low number of retransmissions with a low radiated power level and a simple SIC scheme (see Figure 4). In effect, the additional cancellation provided by the other SIC modules do not bring any significant gain because the number of retransmission is already close to 1 .

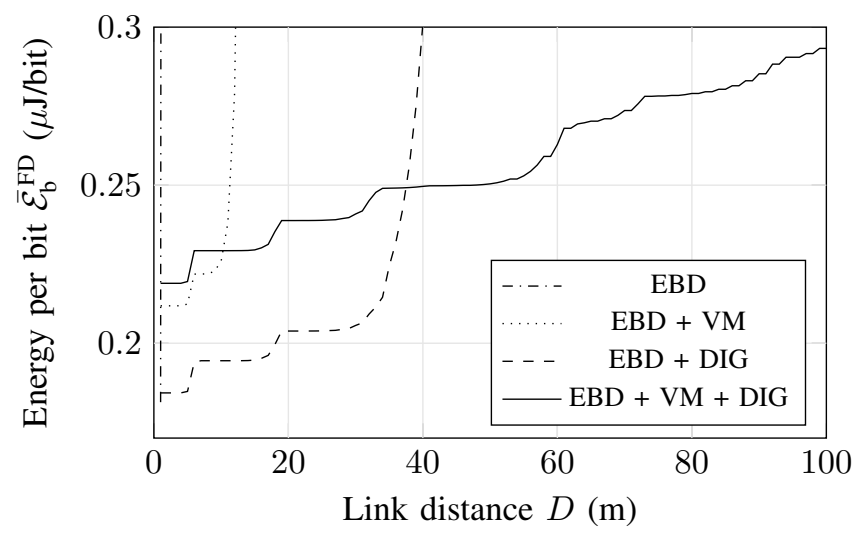

Fig. 3. Energy per bit as a function of link distance for the different SIC configurations

On the other hand, results also show that strong and expensive SIC modules are an energy-efficient solution for large link distances. In effect, the strong path loss introduced by long link distances make it necessary to use high amounts of radiated power. Hence, in this case the SSINR corresponds to (2), being inversely proportional to the cancellation performance of the SIC module. Therefore, a high SIC is needed in order to cope 


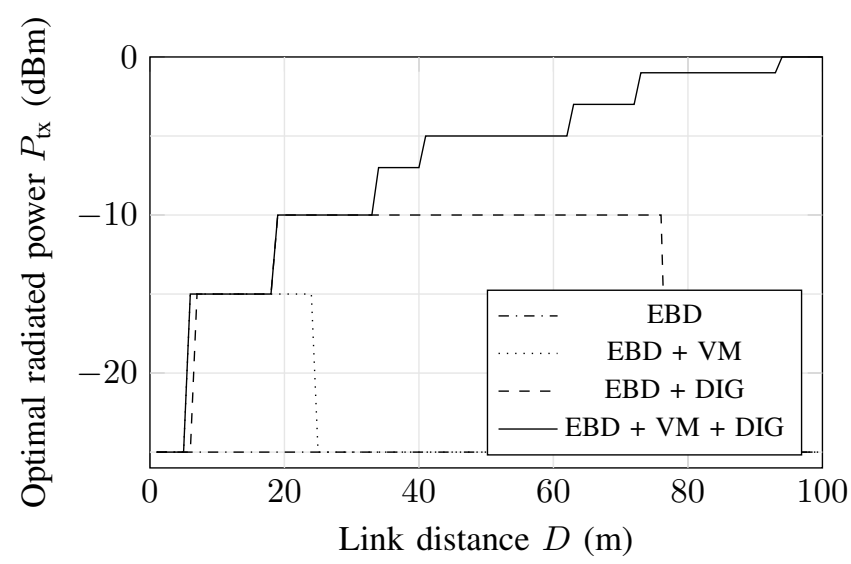

Fig. 4. Optimal transmission power for the different SIC configurations

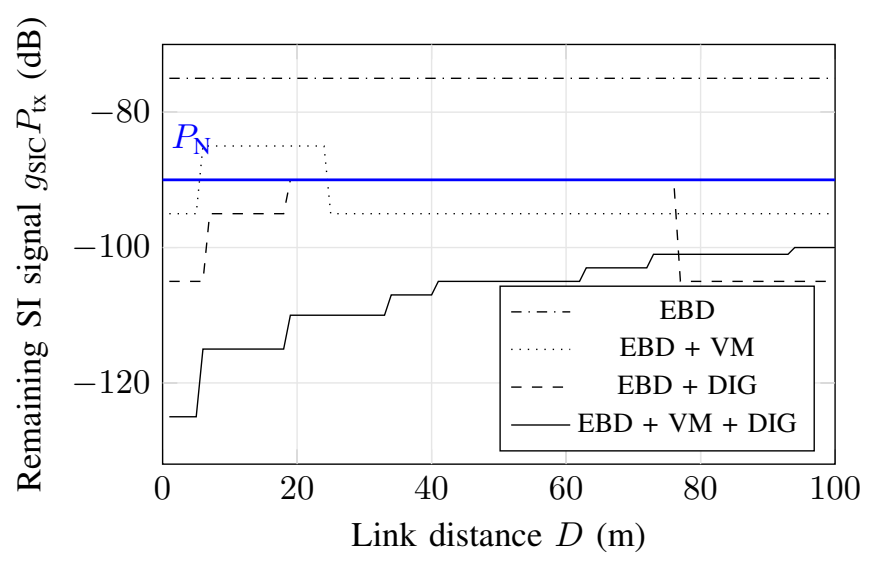

Fig. 5. Optimal remaining SI power for the different SIC configurations

with the high radiated power and hence achieve a low number of retransmissions.

Results also show that the optimal radiated power of all SIC configurations increases until a point where a further increase is no longer beneficial for the SSINR. Interestingly, for the studied scenario, this point corresponds to when the remaining SI signal is just below the noise floor (see Figure 5). In effect, allowing the SI to go above the noise floor is never energyefficient, as this makes the number of retransmissions to grow exponentially. Hence, in the case of a symmetric link $P_{\mathrm{tx}}$ and $g_{\text {SIC }}$ should always be carefully balanced.

It is important to mention that these results hold for symmetrical links, when the SI is linearly proportional to the radiated power. Asymmetrical links have not been included in this work, as they are part of our current ongoing work.

\section{CONCLUSION}

This paper presents a scalable IBFD architecture that adapts the SIC scheme to the link conditions to increase its energy efficiency. In this way, the proposed architecture exploits the trade-off that exists between the SIC performance and the electronic power consumption. The energy efficiency of the proposed architecture is analyzed using an analytical performance model.

Our results showed that the proposed IBFD architecture is able to obtain significant energy savings by turning off some of the SIC modules when performing short-range communications. On the other hand, results show that strong and expensive SIC techniques are only energy-efficient choices for long link distances. For the considered scenarios, it was found that the optimal radiated power increases with the link distance until the SI power reaches the noise floor of the system.

\section{ACKNOWLEDGMENT}

Tom Vermeulen is funded by the "Agency for Innovation by Science and Technology in Flanders (IWT)". This research has been partially funded by the IWT SBO projects SINS and SAMURAI and a Hercules infrastructure grant. The authors would like to thank Bertold Van den Bergh for the fruitful discussions.

\section{REFERENCES}

[1] Cisco, "Cisco visual networking index: Global mobile cisco visual networking index: Global mobile data traffic forecast update, 2014 2019."

[2] [Online]. Available: http://www.gartner.com/newsroom/id/2905717

[3] J. Wells, "Faster than fiber: The future of multi-g/s wireless," Microwave Magazine, IEEE, vol. 10, no. 3, pp. 104-112, May 2009.

[4] D. Bharadia, E. McMilin, and S. Katti, "Full duplex radios," in $A C M$ SIGCOMM Computer Communication Review, vol. 43, no. 4. ACM, 2013, pp. 375-386.

[5] T. Vermeulen and S. Pollin, "Energy-delay analysis of full duplex wireless communication for sensor networks," in Global Communications Conference (GLOBECOM), 2014 IEEE, Dec 2014, pp. 455-460.

[6] B. van Liempd, B. Hershberg, K. Raczkowski, S. Ariumi, U. Karthaus, K.-F. Bink, and J. Craninckx, "A +70dbm iip3 single-ended electricalbalance duplexer in 0.18 um soi cmos," in Solid-State Circuits Conference-(ISSCC), 2015 IEEE International. IEEE, 2015, pp. 32-33.

[7] J. van den Broek, E. Klumperink, and B. Nauta, "A self-interference cancelling receiver for in-band full-duplex wireless with low distortion under cancellation of strong tx leakage," 2015.

[8] J. I. Choi, M. Jain, K. Srinivasan, P. Levis, and S. Katti, "Achieving single channel, full duplex wireless communication," in Proceedings of the sixteenth annual international conference on Mobile computing and networking. ACM, 2010, pp. 1-12.

[9] M. Duarte and A. Sabharwal, "Full-duplex wireless communications using off-the-shelf radios: Feasibility and first results," in Signals, Systems and Computers (ASILOMAR), 2010 Conference Record of the Forty Fourth Asilomar Conference on. IEEE, 2010, pp. 1558-1562.

[10] T. Vermeulen, B. van Liempd, B. Hershberg, and S. Pollin, "Realtime rf self-interference cancellation for in-band full duplex," in $\mathrm{New}$ Frontiers in Dynamic Spectrum Access Networks (DySPAN), 2015 IEEE Symposium on. IEEE, 2015.

[11] F. Rosas and C. Oberli, "Modulation and snr optimization for achieving energy-efficient communications over short-range fading channels," Wireless Communications, IEEE Transactions on, vol. 11, no. 12, pp. 4286-4295, 2012.

[12] F. Rosas, G. Brante, R. D. Souza, and C. Oberli, "Optimizing the code rate for achieving energy-efficient wireless communications," in Wireless Communications and Networking Conference (WCNC), 2014 IEEE. IEEE, 2014, pp. 775-780.

[13] K.-S. Kim and K. Lee, "Low-power and area-efficient fir filter implementation suitable for multiple taps," Very Large Scale Integration (VLSI) Systems, IEEE Transactions on, vol. 11, no. 1, pp. 150-153, 2003.

[14] [Online]. Available: http://www.ti.com/lit/ds/symlink/cc2420.pdf

[15] [Online]. Available: http://www.atmel.com/Images/Atmel-8266MCU_Wireless-ATmega128RFA1_Datasheet.pdf 